\title{
Nuclear spin relaxation due to paramagnetic species in solution: Effect of anisotropy in the zero field splitting tensor
}

\author{
Robert R. Sharp \\ Department of Chemistry, The University of Michigan, Ann Arbor, Michigan 48109
}

(Received 23 November 1992; accepted 4 January 1993)

The NMR (nuclear magnetic resonance) paramagnetic relaxation enhancement (NMR-

PRE) that is produced by paramagnetic solutes in solution has been investigated theoretically with respect to the influence of zero field splitting (zfs) interactions in the electron spin Hamiltonian, in particular with respect to the effects of anisotropy in the zfs tensor. These effects are a physical consequence of the influence of the $z f s$ on the motion of the electron spin vector $\bar{S}$. When the zfs energy is large compared to the Zeeman energy (the $\mathrm{zfs}$ limit), the precessional motion of $\bar{S}$ is quantized in the molecule-fixed coordinate system that diagonalizes the $\mathrm{zfs}$ tensor. The uniaxial portion of the zfs tensor influences the NMRPRE primarily through its influence on the quantization axes of $\bar{S}$; the characteristic behavior of the NMR-PRE under the influence of a uniaxial $\mathrm{zfs}$ has been described in detail previously. Anisotropy in the zfs tensor induces oscillatory motion in $S_{z}$. This motion has a profound influence on the NMR-PRE, the major part of which normally arises from low frequency components of the local magnetic field that are associated with $S_{z}$, rather than from the rapidly precessing local fields that are associated with the transverse components $S_{ \pm}$. For this reason, the NMR-PRE is a sensitive function of $\mathrm{zfs}$ anisotropy, which acts to lower the NMR-PRE below the value that occurs in the uniaxial situation. The magnitude of this effect depends on the ratio $(E / D)$ of the anisotropic and uniaxial zfs parameters, on the reduced dipolar correlation time, and on the location of the nuclear spin in the molecular coordinate frame. A second physical effect of zfs anisotropy on the NMR-PRE arises from a resonance between the electron spin precessional motion in the transverse plane with the precessional motion that is perpendicular to the transverse plane (the latter due to zfs anisotropy). Resonance of these motions, which occurs spin energy levels crossings, gives rise to low frequency transverse components of $\bar{S}$ which result in a resonant increase in the NMR-PRE within a restricted range of $E / D$ ratios.

\section{INTRODUCTION}

Dissolved paramagnetic metal ions in solution often produce profound enhancements of the spin relaxation rates of nuclei on coordinating ligands and on solvent molecules. This effect, which is termed the NMR paramagnetic relaxation enhancement or NMR-PRE, has been widely used to study the chemical and magnetic environment of paramagnetic metal ions in solutions. The classical theory of the NMR-PRE is due to Solomon, ${ }^{1}$ Bloembergen, ${ }^{2}$ and Morgan. ${ }^{2,3}$ This theory (SBM theory), in its original form or modified slightly to describe $g$-tensor anisotropy or to differentiate electron spin $T_{1}$ and $T_{2}$ relaxation times, ${ }^{4}$ is almost universally employed in the analysis of $n \mathrm{mr}$ relaxation data in paramagnetic solutions.

However, SBM theory is a limiting theory in that it assumes that the electron spin Hamiltonian $H_{S} \equiv \hbar \mathscr{H}_{S}$ of the paramagnetic metal ion is the Zeeman Hamiltonian, $\mathscr{H}_{S}=\mathscr{H}_{Z}$, i.e., the theory neglects zero field splitting interactions. The neglect of the zfs Hamiltonian is in many cases physically inappropriate for ions with $S \geqslant 1$, particularly for ions other than $\mathrm{Mn}$ (II) and Gd(III), for which the $\mathrm{zfs}$ is atypically small. In the presence of a large $\mathrm{zfs}$ interaction, the electron spin precessional motion is quantized along molecule-fixed coordinate axes rather than along the external magnetic field axis, as occurs in the
Zeeman limit. This change in quantization axis has important consequences for the behavior of the NMR-PRE. In the zfs limit, the local dipolar magnetic field of the electron spin $S$ is fixed in the molecular coordinate system. In consequence, the magnitude of the NMR-PRE depends strongly on the position of the nuclear spin in the molecular coordinate frame, axial positions being more strongly relaxing than equatorial positions. Two other important physical effects are also associated with the spin requantization phenomenon. These are (1) a change in definition of the reorientational correlation time from $\tau_{R}^{(1)}$ in the $\mathrm{zfs}$ limit to $\tau_{R}^{(2)}$ in the Zeeman limit, where $\tau_{R}^{(l)}$ is the reorientational correlation time for an $l$ th rank molecule-fixed spherical tensor, and (2) effects of level crossings and near level crossings of the energy levels. Each of these physical phenomena gives rise to characteristic qualitative features in the magnetic field dispersion profile of the PRE (i.e., in plots of the magnetic field dependence of the PRE at constant temperature). The features that are characteristic of zfs-limit ions have been discussed systematically for $S=1$ (Ref. 5) and for $S>1 .^{6}$

In previous work I have derived theoretical expressions which parallel the form of the SBM results but describe intramolecular ${ }^{7}$ and intermolecular ${ }^{8}$ relaxation in the $\mathrm{zfs}$ limit. Subsequently, a generalized theory that is appropriate to the intermediate regime was developed. ${ }^{9}$ A principal 
focus of this work, in addition to the development of realistic quantitative theory, has been the development of clear, qualitative physical descriptions of the phenomena that underlie the NMR-PRE for spin systems that are outside the Zeeman limit. Alternate, rather different, theoretical approaches that are based primarily on solutions of the stochastic Liouville equation have also been described. ${ }^{10-26}$ These earlier approaches have for the most part provided a framework for the numerical evaluation of spin relaxation rates, but have not provided much insight concerning the physical situation in the zfs limit.

All previous theoretical work in this area has assumed uniaxial symmetry in the zfs Hamiltonian. It seemed that the effects of zfs anisotropy could be treated as a quantitative correction to the uniaxial case, mathematically more complex but involving no qualitatively new phenomena. In the present study, we show that this is not the case; the presence of anisotropy in the zfs exerts an influence on the motion of the spin vector $\vec{S}$ that has profound consequences for the NMR-PRE. These are associated with the fact that anisotropy in the zfs (unlike the uniaxial portion of the $\mathrm{zfs}$ ) induces oscillatory behavior in the $z$ component of $\vec{S}$. The NMR-PRE is very sensitive to time dependence in $S_{z}$ much more so than to alterations in the precessional time dependence of the transverse components, $S_{ \pm}$. Thus the effects of zfs anisotropy require special consideration.

In the following sections, I first describe the physical effects of zfs anisotropy on the motion of $\bar{S}$ and on the NMR-PRE ( Sec. II). Section III develops formal zfs-limit theory of the NMR-PRE which is based on a spin Hamiltonian that accounts for $z$ fs anisotropy. The fourth section describes the results of theoretical calculations which explore systematically and quantitatively the implications of the theory. And finally, the fifth section discusses limitations of the theory, especially the neglect of effects of the external magnetic field, as well as problems involved with the experimental investigation of these phenomena.

\section{PHYSICAL EFFECTS OF ZFS ANISOTROPY}

To describe the effects of zfs anisotropy on the NMRPRE, we first consider the motion of the spin vector $\bar{S}$ under the influence of the static spin Hamiltonian

$$
\mathscr{H}_{S}=\mathscr{H}_{Z}+\mathscr{H}_{\text {zfs }} \text {, }
$$

where $\mathscr{H}_{Z}$ is the Zeeman Hamiltonian,

$$
\mathscr{H}_{Z}=\mathrm{g} \beta B_{\alpha} S_{0}^{(1)} \text {, }
$$

and $\mathscr{H}_{\text {zfs }}$ is the static zfs Hamiltonian,

$$
\mathscr{H}_{\mathrm{zfs}}=\left(\frac{2}{3}\right)^{1 / 2} \omega_{D} \hat{S}_{0}^{(2)}+\omega_{E}\left[\hat{S}_{+2}^{(2)}+\hat{S}_{-2}^{(2)}\right]
$$

In Eq. (1b), $\mathbf{g}$ is the electron spin $\mathbf{g}$ value, $\beta$ is the Bohr magneton, and $B_{0}$ is the static magnetic field strength. In Eq. (1c), $\omega_{D}$ and $\omega_{E}$ are the zero field splitting parameters $D$ and $E\left(\mathrm{~cm}^{-1}\right)$ expressed in $\mathrm{rad} \mathrm{s}^{-1}$. The zfs Hamiltonian in Eq. (1c) includes terms that are quadratic in the electron spin operators; for $S \geqslant 2$, higher order terms are in general present. The spin variables in Eqs. (1) are first and second rank spherical tensor operators constructed from the component operators of $S,{ }^{27}$

$$
\begin{aligned}
S_{ \pm 1}^{(1)} & =\mp 2^{-1} S_{ \pm}, \\
S_{0}^{(1)} & =S_{z} \\
S_{0}^{(2)} & =6^{-1 / 2}\left[3 S_{z}^{2}-S(S+1)\right] \\
& =6^{-1 / 2}\left[2 S_{0}^{(1)} S_{0}^{(1)}+S_{+1}^{(1)} S_{-1}^{(1)}+S_{-1}^{(1)} S_{+1}^{(1)},\right. \\
S_{ \pm 2}^{(2)} & =2^{-1} S_{ \pm} S_{ \pm}=S_{ \pm 1}^{(1)} S_{ \pm 1}^{(1)} .
\end{aligned}
$$

In the Zeeman limit $\left(\mathscr{H}_{Z} \gg \mathscr{H}_{\text {zfs }}\right)$, the motion of $\bar{S}$ is familiar: the spin Hamiltonian $\mathscr{H}_{S}$ equals the Zeeman Hamiltonian $\mathscr{H}_{Z}$, which is diagonal in the laboratory coordinate frame $(x, y, z)$. Since $\left[\mathscr{H}_{S}, S_{z}\right]=0, S_{z}$ is time invariant, $\left\langle S_{Z}\right\rangle=m_{s}$, and the precessional motion of $\bar{S}$ occurs entirely in the transverse plane.

In the zfs limit $\left(\mathscr{H}_{\text {zfs }} \gg \mathscr{H}_{Z}\right)$, the precessional motion of $\bar{S}$ is quantized along the molecule-fixed coordinate axes $(\hat{x}, \hat{y}, \hat{z})$ which diagonalize the zfs tensor. [In the following and in Eq. (1c), variables that are defined with respect to the molecule-fixed coordinate frame are denoted by a superscripting karat (i.e., $\hat{x}$ ), and variables defined in the laboratory frame are written without a karat.] For simplicity, we restrict consideration to $S=1$, for which the matrix representation of $\mathscr{H}_{\mathrm{zfs}}$, written in the molecule-fixed coordinate frame, can be written

$$
\begin{aligned}
\mathscr{H}_{\mathrm{zfs}}= & \left(\frac{2}{3}\right)^{1 / 2} \omega_{D} 6^{-1 / 2}\left(\begin{array}{ccc}
1 & 0 & 0 \\
0 & -2 & 0 \\
0 & 0 & 1
\end{array}\right) \\
& +\omega_{E}\left\{\left(\begin{array}{lll}
0 & 0 & 1 \\
0 & 0 & 0 \\
0 & 0 & 0
\end{array}\right)+\left(\begin{array}{lll}
0 & 0 & 0 \\
0 & 0 & 0 \\
1 & 0 & 0
\end{array}\right)\right\} .
\end{aligned}
$$

When the zfs tensor is uniaxial $(E=0),\left[\mathscr{H}_{\mathrm{zfs}}, \hat{S}_{\mathrm{z}}\right]=0$, and $\hat{S}_{z}$ is a constant of the motion. As in the Zeeman limit, the precessional motion of $\bar{S}$ occurs entirely in the transverse plane, although in the zfs limit this is the transverse plane of the molecular coordinate frame rather than the laboratory frame. $\hat{S}_{z}$ is time invariant with $\left\langle\hat{S}_{z}\right\rangle=m_{S}$.

In the presence of zfs anisotropy, $\mathscr{H}_{\text {zfs }}$ is not diagonal, and $\hat{S}_{z}$, like $\hat{S}_{x}, \hat{S}_{y}$, undergoes precessional motion in the molecular frame. The spin variables $\hat{S}_{ \pm 2}^{(2)}$ in the anisotropic term of $\mathscr{H}_{\text {zfs }}$ connect states with $\Delta m_{S}= \pm 2$. Thus for $S=1$, the anisotropy has no effect on the $m_{S}=0$ state, but mixes the $m_{S}= \pm 1$ states. The effect of this on the motion of $\hat{S}_{z}$ can be illustrated by plotting the time evolution of the density matrix, $\hat{\rho}(t)$, under the influence of the static Hamiltonian $\mathscr{H}_{\text {zfs }}$. This has been done for $S=1$ by integrating numerically the equation of motion of $\hat{\rho}(t)$,

$$
\frac{d \hat{\rho}(t)}{d t}=-i\left[\mathscr{H}_{\mathrm{zf},}, \hat{\rho}(t)\right],
$$

using selected values of $\omega_{D}$ and $\omega_{E}$. The initial state was taken to be $\hat{\rho}_{1,1}(0)=1, \hat{\rho}_{0,0}(0)=0$, and $\hat{\rho}_{-1,-1}(0)=0$. 


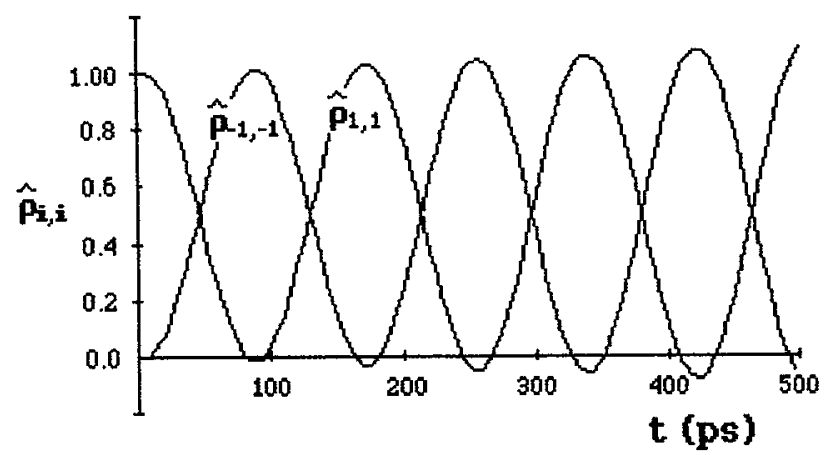

FIG. 1. Time evolution of the diagonal elements $\hat{\rho}_{1,1}$ and $\hat{\rho}_{-1,-1}$ of the density matrix of an $S=1$ spin system under the influence of an anisotropic zero field splitting Hamiltonian. The time evolution of $\hat{\rho}$ was calculated numerically from the equation of motion of the density matrix assuming initial conditions $\hat{\rho}_{1,1}(0)=1, \hat{\rho}_{0,0}(0)=\hat{\rho}_{-1,-1}(0)=0$. The assumed zfs parameters were $D=1 \mathrm{~cm}^{-1}$ and $E / D=0.1$, and the step size of the calculation was $0.5 \mathrm{ps}$.

Thus the spin system is assumed to have been prepared in the $m_{S}=+1$ state at $t=0$. Under the influence of $\mathrm{zfs}$ anisotropy, the diagonal elements $\hat{\rho}_{1,1}$ and $\hat{\rho}_{-1,-1}$ oscillate sinusoidally in time with angular frequency $2 \omega_{E}$, while the $\hat{\rho}_{0,0}$ element remains constant. The time dependence of $\hat{\rho}_{1,1}$ and $\hat{\rho}_{-1,-1}$ are illustrated in Figs. 1 and 2, which show results for $D=1 \mathrm{~cm}^{-1}$, with $E / D=0.1$ (Fig. 1) and $E / D$ $=0.05$ (Fig. 2). (The fact that the $\rho_{i, i}$ exceed +1 and fall below zero at long time in Figs. 1 and 2 is an artifact of the finite step size of the calculation.) The corresponding time correlation function, $G_{z}(t)=\left\langle\hat{S}_{z}(0) \cdot \hat{S}_{z}(t)\right\rangle$, is likewise an oscillating function of time with angular frequency $2 \omega_{E}$ (Fig. 3).

The influence of these precessional phenomena on the NMR-PRE can be summarized as follows. The largest part of the PRE normally results from the low frequency motions of $\bar{S}$, since these generally give rise to local fields with relatively large Fourier components at the nuclear precession frequency, $\omega_{I}$. In the Zeeman limit, as well as in the zfs limit with uniaxial symmetry, $S_{z}$ (or $\hat{S}_{z}$ ) and the asso-

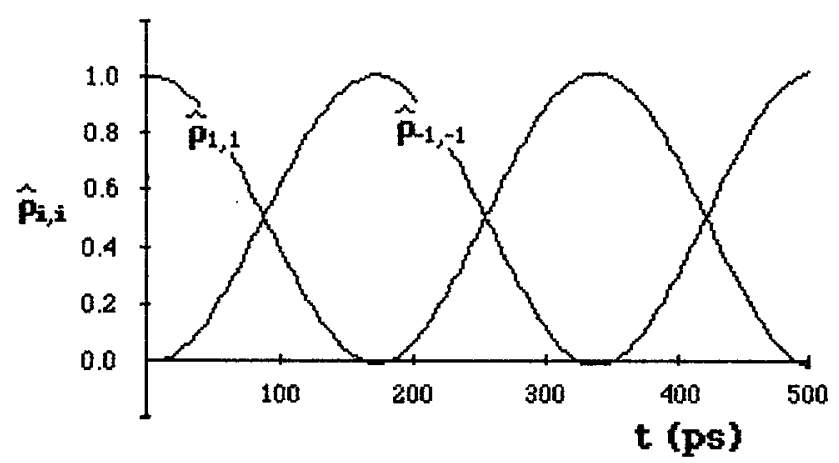

FIG. 2. Time evolution of the diagonal elements $\hat{\rho}_{1,1}$ and $\hat{\rho}_{-1,-1}$ of the density matrix of an $S=1$ spin system under the influence of an anisotropic zero field splitting Hamiltonian. The conditions are the same as for Fig. 1 except $E / D=0.05$.

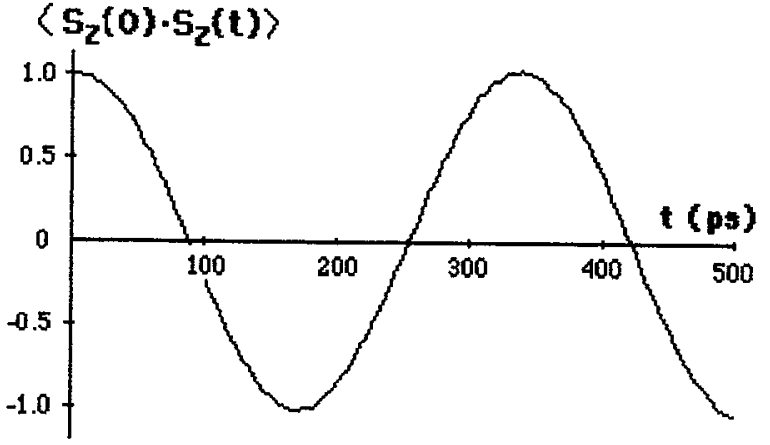

FIG. 3. The time correlation function $G_{z}(t)=\operatorname{Tr}\left\{\hat{S}_{z}(0) \cdot \hat{S}_{z}(t)\right\}$ corresponding to the density matrix $\hat{\rho}(t)$ of Fig. 2 .

ciated components of the local dipolar magnetic field that arise from $S_{z}$ (or $\hat{S}_{z}$ ) are static with respect to spin precession. (Although $S_{z}$ does not precess, it still undergoes stochastic time dependence due to electron spin relaxation and molecular reorientation.) The motion of $S_{z}$ appears in the theory of the NMR-PRE in a spectral density function which is, in the zfs limit,

$$
\begin{aligned}
j_{z}(\omega) & =\int_{0}^{\infty}\left\langle\hat{S}_{z}(0) \cdot \hat{S}_{z}(t)\right\rangle \exp \left( \pm i \omega_{I} t\right) d t, \\
& =\frac{S(S+1)}{3} \int_{0}^{\infty} \exp \left(-t / \tau_{c}\right) \exp \left( \pm i \omega_{I} t\right) d t, \\
& =\frac{S(S+1)}{3} j\left(\omega_{I}\right),
\end{aligned}
$$

with $j(\omega)=\tau_{d}\left(1+\omega^{2} \tau_{c}^{2}\right)$. In Eq. (5b), the effects of the stochastic time dependence of $\hat{S}_{z}$ are described as an exponential decay with time constant $\tau_{c}$.

The transverse components of $\bar{S}$ oscillate due to spin precession. Their contributions to the NMR-PRE are proportional to spectral density terms $j\left(\omega_{\mu} \pm \omega_{I}\right)$, where the $\omega_{\mu}$ are transition frequencies of the electronic spin system. In the Zeeman limit, these are the terms $j\left(\omega_{S} \pm \omega_{I}\right)$ in SBM theory. When $\omega_{\mu} \tau_{c}>1$ (a common situation in the zfs limit), these terms are much smaller than $j\left(\omega_{I}\right)$. Thus the "low frequency" terms described by Eqs. (5) usually comprise the largest part of $R_{1 p}$.

The principal physical effect of zfs anisotropy is to produce sinusoidal time dependence in the time correlation function $\left\langle\hat{S}_{z}(0) \cdot \hat{S}_{z}(t)\right\rangle$ in Eq. (5a). This results in the replacement of $j\left(\omega_{I}\right)$ in Eq. (5c) by a higher frequency term which, for $S=1$, is $j\left(\omega_{I} \pm 2 \omega_{E}\right) . j\left(\omega_{I} \pm 2 \omega_{E}\right)$ is in general smaller than $j\left(\omega_{I}\right)$, significantly so when $2 \omega_{E} \tau_{c}$ $>1$. Anisotropy in the zfs tensor thus acts to reduce the NMR-PRE below the value that would occur for $E / D=0$. Its effects are important when $\hat{S}_{z}$ undergoes significant precessional motion on the time scale of $\tau_{c}$, i.e., when $2 \omega_{E} \tau_{c}$ $>1$.

In addition to its effect on $\left\langle\hat{S}_{z}(0) \cdot \hat{S}_{z}(t)\right\rangle$, the zfs anisotropy also affects the motion of the transverse components of $\bar{S}$ in a manner which significantly influences the 
NMR-PRE. This second phenomenon is reflected in the behavior of the matrix elements $\left\langle\hat{S}_{ \pm}(0) \cdot \hat{S}_{\mp}(t)\right\rangle$ and results from a resonance between the precessional motion due to $D$ and that due to $E$. Resonance between these precessions produces low frequency components in the transverse magnetization which can provide a significant contribution to the NMR-PRE. This phenomenon has the functional form of a resonance and acts to enhance the NMR-PRE in a relatively narrow range of $E / D$ ratios. Its properties are described at greater length below.

\section{THEORETICAL}

In this section, anisotropic zfs-limit expressions describing the paramagnetic relaxation increment due to electron-nuclear magnetic dipole coupling are derived. The precessional motion of the electron spin is governed by the Hamiltonian defined in Eq. (1c) above. The electronnuclear magnetic dipole-dipole coupling Hamiltonian can be written in spherical tensor form as

$$
\mathscr{H}_{\mathrm{d}-\mathrm{d}}=\frac{\kappa}{r^{3}} \sum_{q} 3^{-1 / 2}(-1)^{1-q} I_{q}^{(1)} F_{-q}^{(1)},
$$

where

$$
\kappa=-30^{1 / 2} \gamma_{I} \mathrm{~g} \beta_{0}\left(\frac{\mu_{0}}{4 \pi}\right)
$$

where $r$ is the interspin $I-S$ distance, $\gamma_{I}$ is the proton magnetogyric ratio, and $\mu_{0}$ is the magnetic permeability of free space. $I_{q}^{(1)}$ are the nuclear spin variables in spherical tensor form [see Eqs. (2a) and (2b)]. The lattice variables are contained in the spherical tensor $F^{(1)}$, which is a function of the spatial variables $(\phi, \vartheta)$ and the electron spin variables $S_{q}^{(1)}$. $F^{(1)}$ can be constructed ${ }^{27}$ by contracting the first rank spherical tensor of the spin variables with the second rank spherical tensor of the spatial variables, $C^{(2)}(\phi, \vartheta)$,

$$
F^{(1)}=\left\{S^{(1)} \otimes C^{(2)}\right\}^{(1)} \text {. }
$$

The components of $C^{(2)}$ are

$$
C_{m}^{(2)}(\vartheta, \phi)=\left(\frac{4 \pi}{5}\right)^{1 / 2} Y_{2, m}(\vartheta, \phi),
$$

where the $Y_{2, m}(\vartheta, \phi)$ are the usual spherical harmonics. Explicit forms of the components of $F^{(1)}$ are

$$
F_{m}^{(1)}=\sum_{p=-1}^{1} c_{m, p} S_{p}^{(1)} C_{m-p}^{(2)}(\vartheta, \phi),
$$

where

$$
\begin{aligned}
& c_{1,1}=c_{-1,-1}=10^{-1 / 2}, \\
& c_{0.1}=c_{0,-1}=-c_{1,0}=-c_{-1,0}=\left(\frac{3}{10}\right)^{1 / 2}, \\
& c_{1 .-1}=c_{-1,1}=\left(\frac{3}{5}\right)^{1 / 2}, \\
& c_{0,0}=-\left(\frac{2}{5}\right)^{1 / 2} .
\end{aligned}
$$

In the zfs limit, the precessional motion of the electron spin is quantized with respect to molecular coordinate axes, and it is convenient in this situation to express the lattice functions, i.e, the components of $F^{(1)}$, in the molecular coordinate frame which diagonalizes the zfs tensor. This transformation is effected by the Wigner rotation matrix elements,

$$
F_{q}^{(1)}=\sum_{q^{\prime}} \hat{F}_{q^{\prime}}^{(1)} \mathscr{D}_{q, q^{\prime}}^{(1)}(\alpha \beta \gamma),
$$

where $\alpha \beta \gamma$ are the Euler angles which rotate the laboratory frame to the molecular frame.

The density matrix theory of nuclear spin relaxation gives, for the dipolar paramagnetic relaxation increment,

$$
\begin{aligned}
R_{1 p, \mathrm{dip}}= & -\frac{\kappa^{2}}{r^{6}} 3^{-1}\left\{\int _ { 0 } ^ { \infty } d t \left(\left\langle F_{-1}^{(1)}(t) \cdot F_{1}^{(1)}(0)\right\rangle e^{i \omega_{I} t}\right.\right. \\
& \left.\left.+\left\langle F_{1}^{(1)}(t) \cdot F_{-1}^{(1)}(0)\right\rangle e^{-i \omega_{I} t}\right)\right\}_{\mathrm{sp} \mathrm{av}},
\end{aligned}
$$

where the curly brackets represent an average over molecular orientations in the laboratory frame (i.e., over the Euler angles $\alpha \beta \gamma$ ), and the square brackets indicate a trace over the electron spin operators. After transforming the components of $F^{(1)}$ to the molecular coordinate frame, the products in the integrand of Eq. (13) become

$$
\begin{aligned}
& \left\langle F_{ \pm 1}^{(1)}(t) \cdot F_{\mp 1}^{(1)}(0)\right\rangle e^{\mp i \omega_{f^{t}}} \\
& =\sum_{q^{\prime}, q^{\prime \prime}}\left\langle F_{q^{\prime}}^{(1)}(t) \cdot \hat{F}_{q^{\prime \prime}}^{(1)}(0)\right\rangle \mathscr{D}_{q^{\prime}, \pm 1}^{(1)}\left(\alpha^{\prime} \beta^{\prime} \gamma^{\prime}, t\right) \\
& \quad \times \mathscr{D}_{q^{\prime}, \mp 1}^{(1)}(\alpha \beta \gamma ; 0) e^{\mp i \omega_{I^{t}},}
\end{aligned}
$$

where the transformed tensor components of $\hat{F}^{(1)}$ are given by Eq. (10)

$$
\hat{F}_{m}^{(1)}=\sum_{p=-1}^{1} c_{m, p} \hat{S}_{p}^{(1)} \hat{C}_{m-p}^{(2)}(\Theta, \Phi) .
$$

In the transformed expression Eq. (15), the spin operators $\hat{S}_{p}^{(1)}$ and the spatial functions $\hat{C}_{q}^{(2)}(\Theta, \Phi)$ are expressed relative to molecular axes. The functions $\hat{C}_{m-p}^{(2)}(\Theta, \Phi)$ describe the (fixed) orientation of the $I-S$ vector in the molecular coordinate frame in terms of its polar angles $\Theta, \Phi$. When the precessional motion of the electron spin is in the zfs limit, $\mathscr{H}_{S}$ is given by Eq. (1c), and the precessional motion is independent of molecular orientation. Thus in the zfs limit the components of the lattice tensor $\hat{F}^{(1)}$ contain no explicit or implicit dependence on the Euler angles $\alpha \beta \gamma$. The spatial average over molecular orientations can then be evaluated analytically by spatially averaging the products of the rotation matrix elements. For a molecule undergoing classical isotropic reorientational diffusion, the spatial average is

$$
\begin{aligned}
& \left\{\mathscr{D}_{q,-1}^{(1)}\left(\alpha^{\prime} \beta^{\prime} \gamma^{\prime} ; t\right) \mathscr{D}_{q^{\prime},+1}^{(1)}(\alpha \beta \gamma ; 0)\right\}_{\mathrm{sp} \mathrm{av}} \\
& \quad=(-1)^{q+1}\left|\mathscr{D}_{q,-1}^{(1)}\right|^{2} \exp \left[-t / \tau_{R}^{(1)}\right] \delta_{q^{\prime},-q}, \\
& \quad=(-1)^{q+1} 3^{-1} \exp \left[-t / \tau_{R}^{(1)}\right] \delta_{q^{\prime},-q}
\end{aligned}
$$


where $\tau_{R}^{(1)}$ is the reorientational correlation time of a first rank molecule-fixed tensor.

Equations (14), (15), and (16) give

$$
\begin{aligned}
\sum_{q}\left\langle\hat{F}_{q}^{(1)}(t) \hat{F}_{-q}^{(1)}(0)\right\rangle(-1)^{q+1} \frac{\exp \left[-t / \tau_{R}^{(1)}\right]}{3} e^{\mp i \omega_{I^{t}}} \\
=3^{-1} \exp \left[-t / \tau_{R}^{(1)}\right] e^{\mp i \omega_{I^{t}}} \sum_{p, p^{\prime}}\left\langle S_{p}^{(1)}(t) \hat{S}_{p^{\prime}}^{(1)}(0)\right\rangle \\
\quad \times \sum_{q=-1}^{1}(-1)^{q+1} c_{q, p} \mathcal{C}_{-q, p^{\prime}} \hat{C}_{q-p}^{(2)} \hat{C}_{-q-p^{\prime}}^{(2)}
\end{aligned}
$$

In the sum over $p, p^{\prime}$, certain terms are identically zero due to the symmetry of $\mathscr{H}_{\mathrm{zfs}}$. This point is discussed in the Appendix, where it is shown that nonzero terms for an isotropic zfs tensor are those with $p+p^{\prime}=0$, and for an anisotropic zfs tensor, those with $p+p^{\prime}$ even.

As has been shown previously, ${ }^{9}$ the time dependence of the trace $\left\langle S_{p}^{(1)}(t) \hat{S}_{p^{\prime}}^{(1)}(0)\right\rangle$ can be expressed in a form that is suitable for numerical calculations when it is evaluated in the eigenbasis of $\mathscr{H}_{\text {zfs. }}$. In this basis, assuming the high temperature limit for the $S$ spin,

$$
\begin{aligned}
\operatorname{Tr}\left\{\hat{S}_{p}^{(1)}(t) \hat{S}_{p^{\prime}}^{(1)}(0)\right\}= & (2 S+1)^{-1} \sum_{\mu, v} e^{i\left(\omega_{\mu}-\omega_{\nu}\right) t} e^{-t / \tau_{S}} \\
& \times\left\langle\mu\left|\hat{S}_{p}^{(1)}\right| v\right\rangle\left\langle\nu\left|\hat{S}_{p^{\prime}}^{(1)}\right| \mu\right\rangle,
\end{aligned}
$$

where $\omega_{\mu}, \omega_{v}$ are eigenvalues and $|\mu\rangle,|v\rangle$ eigenvectors of $\mathscr{H}_{\mathrm{zfs}} \cdot \tau_{S}$ is the electron spin relaxation time, assumed to be isotropic with respect to molecular axes. When $S>1$, the use of a single parameter $\tau_{S}$ is an approximation since electron spin relaxation is then a multiexponential pro$\operatorname{cess}^{28}$. For an isotropic zfs tensor $(E=0), \mathscr{H}_{S}$ is diagonal, and the matrix elements can be written in a simple analytical form. ${ }^{7}$ In the more general situation where $E \neq 0$, the matrix elements of $\hat{S}_{p}^{(1)}$ must be evaluated numerically by diagonalizing $\mathscr{H}_{\mathrm{zfs}}$.

After inserting Eq. (17) into Eq. (13), expanding the sum, and evaluating the spatial average according to Eqs. (16), $R_{1 p \text {,dip }}$ can be written

$$
R_{1 p, \text { dip }}=4\left(\frac{\gamma_{I} g \beta}{r^{3}}\right)^{2}\left(\frac{\mu_{0}}{4 \pi}\right)^{2}\left\{\mathbb{T}_{1}+\mathbb{T}_{2}+\left(\mathbb{T}_{3}+\mathbb{T}_{3}^{*}\right)\right\}
$$

with

$$
\begin{aligned}
\mathbb{T}_{1}= & {\left[\frac{2}{3} \hat{C}_{0}^{(2)} \hat{C}_{0}^{(2)}-\hat{C}_{+1}^{(2)} \hat{C}_{-1}^{(2)}\right] \hat{m}_{0,0}^{( \pm)}, } \\
= & 3^{-1}\left[1+\hat{P}_{2}(\cos \Theta)\right] \hat{m}_{0,0}^{( \pm)}, \\
\mathbb{T}_{2}= & {\left[6^{-1} C_{0}^{(2)} C_{0}^{(2)}-2^{-1} C_{+1}^{(2)} C_{-1}^{(2)}+C_{+2}^{(2)} C_{-2}^{(2)}\right] } \\
& \times\left[\hat{m}_{1,-1}^{( \pm)}+\hat{m}_{-1,1}^{( \pm)}\right], \\
= & 3^{-1}\left[1-2^{-1} P_{2}(\cos \Theta)\right]\left[\hat{m}_{1,-1}^{( \pm)}+\hat{m}_{-1,1}^{( \pm)}\right], \\
\mathbb{T}_{3}= & {\left[C_{+1}^{(2)} C_{+1}^{(2)}-4 \cdot 6^{-1 / 2} C_{0}^{(2)} C_{+2}^{(2)}\right] \hat{m}_{-1,-1}^{( \pm)} . }
\end{aligned}
$$

The functions $\hat{m}_{q, q^{\prime}}^{( \pm)}$are defined

$$
\begin{aligned}
\hat{m}_{q, q^{\prime}}^{( \pm)} \equiv & \int_{0}^{\infty} d t \exp \left( \pm i \omega_{I} t\right) \exp \left[-t / \tau_{R}^{(1)}\right] \\
& \times \operatorname{Tr}\left\{\hat{S}_{q}^{(1)}(t) \hat{S}_{q^{\prime}}^{(1)}(0)\right\}
\end{aligned}
$$

Using Eq. (18) to evaluate the trace gives

$$
\begin{aligned}
\hat{m}_{p, p^{\prime}}^{( \pm)}= & \int_{0}^{\infty} d t \exp \left( \pm i \omega_{I} t\right) \exp \left[-t / \tau_{R}^{(1)}\right] \exp \left(-t / \tau_{S}\right) \\
& \times(2 S+1)^{-1} \sum_{\mu, \nu}\left\langle\mu\left|\hat{S}_{p}^{(1)}\right| v\right\rangle \\
& \times\left\langle v\left|\hat{S}_{p^{\prime}}^{(1)}\right| \mu\right\rangle \exp \left[i\left(\omega_{\mu}-\omega_{\nu}\right) t\right], \\
= & (2 S+1)^{-1} \sum_{\mu, v}\left\langle\mu\left|\hat{S}_{p}^{(1)}\right| v\right\rangle\left\langle v\left|\hat{S}_{p^{\prime}}^{(1)}\right| \mu\right\rangle \\
& \times j\left(\omega_{\mu}-\omega_{\nu} \pm \omega_{I}\right),
\end{aligned}
$$

where $\tau_{c}^{-1}=\tau_{S}^{-1}+\left[\tau_{R}^{(1)}\right]^{-1}$.

Equation (19) represents a generalization of the zfslimit result of Ref. 7, which was derived for a uniaxial zfs tensor. When $E=0$ in Eq. (1c), $\mathbb{T}_{3}=0, \mathscr{H}_{S}$ is diagonal, and the functions $\hat{m}_{p, p^{\prime}}^{( \pm)}$can be written in analytical form. The expression for $R_{1 p \text {,dip }}$ then reduces to

$$
\begin{aligned}
R_{1 p, \mathrm{dip}}= & \frac{4}{3}\left(\frac{\gamma_{I} \mathrm{~g} \beta}{r^{3}}\right)^{2}\left(\frac{\mu_{0}}{4 \pi}\right)^{2}\left\{\frac{S(S+1)}{3}\left[1+\hat{P}_{2}(\cos \Theta)\right] j\left(\omega_{I}\right)\right. \\
& +2(S+1)^{-1}\left[1-2^{-1} \hat{P}_{2}(\cos \Theta)\right] \\
& \left.\times \sum_{\mu}\left|c_{S, \mu}^{+}\right|^{2} j\left(\omega_{\mu}\right)\right\}
\end{aligned}
$$

which is identical to the previous zfs-limit result. When the zfs tensor is anisotropic, $R_{1 p}$ can be evaluated numerically from Eqs. (19)-(23)

\section{THEORETICAL RESULTS}

Calculations illustrating the effect of zfs anisotropy on the NMR-PRE have been carried out for an electron spin $S=1$, and the results are displayed in Figs. (4)-(12). Figure 4 shows the variation of $R_{1 p, \text { dip }}$ as a function of the $E / D$ ratio for a nuclear spin $I$ located in an axial $(\Theta=0)$ and equatorial $(\Theta=\pi / 2)$ positions in the molecular coordinate frame. The azimuthal angle $\Phi$ was taken to be zero. To suppress the effects of scaling factors such as the interspin distance $r_{I S}$, the results are plotted as the ratio of $R_{1 p}(E /$ $D$ ), calculated at the specified $E / D$ ratio, over $R_{1 p}(0)$, calculated for $E / D=0$. The results depend on the dimensionless quantity $\omega_{D} \tau_{c}$, the reduced dipolar correlation time, which was taken to be 11.7.

With increasing $E / D, R_{1 p}$ declines in magnitude, this decline being interrupted by a resonant local maximum in $R_{1 p}$ near $E / D=1 . R_{1 p}$ falls off somewhat more rapidly for $\Theta=0$ than for $\Theta=\pi / 2$. As described above, the physical origin of this decreasing phase is precessional motion in 


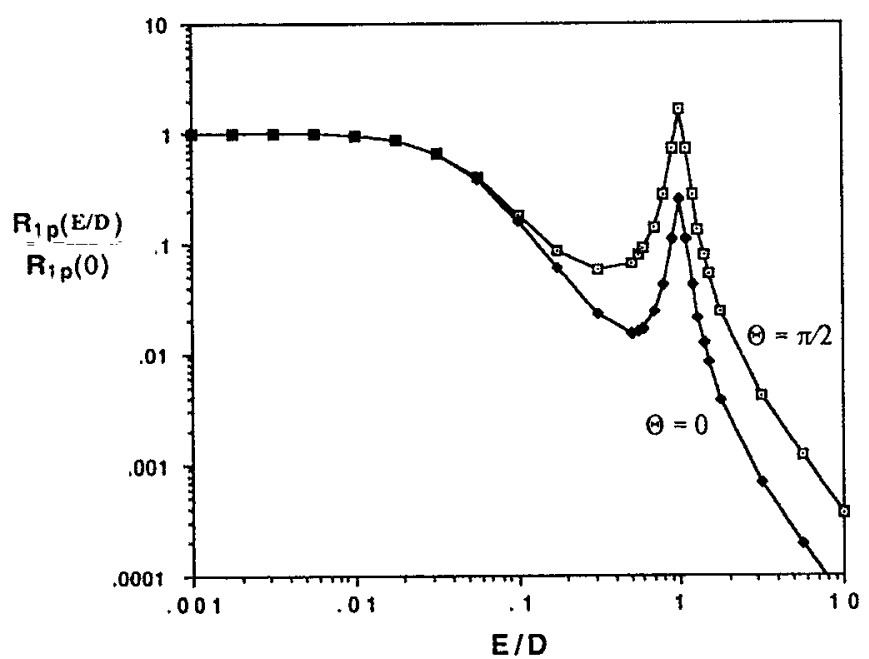

FIG. 4. The NMR paramagnetic relaxation enhancement for a spin $S=1$, evolving under the anisotropic zfs Hamiltonian of Eq. (1c), as a function of $E / D$ ( $E$ and $D$ are zfs parameters). Results have been normalized to the calculated $R_{1 \rho}$ at $E / D=0$. The reduced dipolar correlation time $\omega_{D} \tau_{c}$ $=11.7 . \Theta$ and $\Phi$ are polar angles which specify the nuclear position in the molecular coordinate frame that diagonalizes the zfs tensor. $\Theta=0$ or $\pi / 2$, and $\Phi=0$.

$\hat{S}_{z}(t)$ that is induced by the zfs anisotropy. This motion becomes significant when $2 \omega_{E} \tau_{c} \gtrsim 1$; the point $2 \omega_{E} \tau_{c}=1$ is denoted by the arrow in Fig. 6 .

The resonant increase in $R_{1 p}$, which interrupts the decreasing phase at $E / D=1$, coincides with a level crossing of the spin system. This is shown in Fig. 5, where the energy levels for an $S=1$ spin system, subject to the quadratic zfs Hamiltonian of Eq. (3), are plotted vs $E / D$. The effect of the level crossings appears in the theory of Sec. III in the functions $\hat{m}_{ \pm 1, \mp 1}^{( \pm)}$, which play an analogous role in the generalized theory to the spectral density functions $j\left(\omega_{S} \pm \omega_{I}\right)$ in Zeeman-limit theory and the functions $j\left(\omega_{\mu}\right.$ $\left.\pm \omega_{l}\right)$ in the uniaxial zfs-limit theory. The functions $\hat{m}_{ \pm 1, \mp 1}^{( \pm)}$, like the functions $j\left(\omega_{\mu} \pm \omega_{I}\right)$, contain energy denominators which become small in the vicinity of $\Delta m_{S}$ $= \pm 1$ level crossings. An alternative, somewhat more mechanistic, view of this phenomenon is as a resonance of the precessional motions about $\hat{z}$ ( $D$-type precession) and about the axis of $E$-type precession in the perpendicular plane. At resonance, the two precessional motions interfere

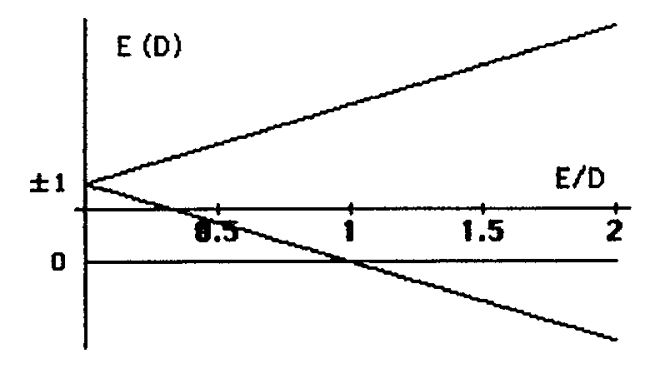

FIG. 5. Energy levels of a spin $S=1$ under the influence of an anisotropic quadratic zero field splitting Hamiltonian. The Zeeman energy is zero.

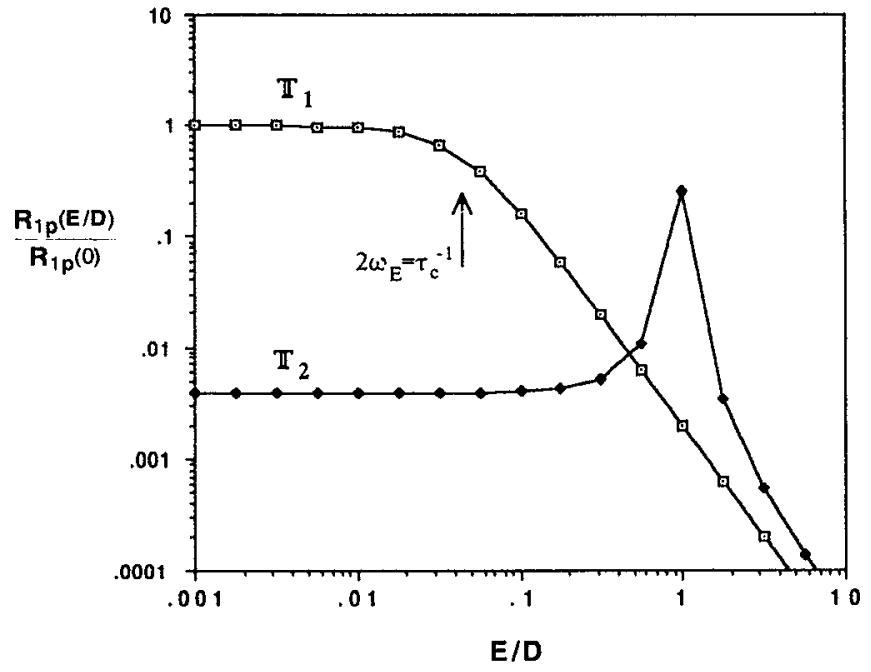

FIG. 6. Contributions to the dipolar NMR-PRE for $S=1$ arising from the motion of $G_{z}(t)=\operatorname{Tr}\left\{\hat{S}_{z}(0) \cdot \hat{S}_{z}(t)\right\}\left(\mathrm{T}_{1}\right)$ and from the motion of $G_{ \pm}(t)=\operatorname{Tr}\left\{\hat{S}_{ \pm}(0) \cdot \hat{S}_{\mp}(t)\right\}\left(\mathbb{T}_{2}\right)$. The calculated relaxation enhancements assume $\Theta=0, \Phi=0, \omega_{D} \tau_{c}=11.7$, and are normalized to $R_{1 p}$ at $E / D=0$, where $D$ is the uniaxial and $E$ the anisotropic zfs parameters.

in a way which produces a time-invariant component of the projection $\hat{S}_{x y}$ of $\bar{S}$ in the transverse plane. Thus the resonance phenomenon is a property specifically of the terms $\mathbb{T}_{2}$ and $\mathbb{T}_{3}$ of Eq. (19), which describes the motion of the transverse components of $\bar{S}$. This is illustrated in Fig. 6, which shows calculations of $\mathbb{T}_{1}$ and $\mathbb{T}_{2}$ plotted as a function of $E / D$ for $\Theta=0, \Phi=0$ with $\omega_{D} \tau_{S}=11.7$. The term $\mathbb{T}_{3}$ vanishes at $\Phi=0$ and thus is not shown. The plotted values of $\mathbb{T}_{1}$ and $\mathbb{T}_{2}$ are normalized to $\mathbb{T}_{1}(0)$, which is the value at $E / D=0$. The calculations of Fig. 6 confirm that the contribution to $R_{1 p}$ of $\left\langle\hat{S}_{z}(0) \cdot \hat{S}_{z}(t)\right\rangle$ falls off monotonically with $E / D$ and that the resonance originates entirely in the behavior of $\left\langle\hat{S}_{ \pm}(0) \cdot \hat{S}_{\mp}(t)\right\rangle$.

Figure 7 shows the overall dependence of the NMRPRE on the reduced correlation time $\omega_{D} \tau_{c}$. As the reduced correlation time lengthens, the normalized enhancements become increasingly sensitive to the effects of zfs anisotropy. In line with the above discussion, this is due physically to the fact that longer $\omega_{D} \tau_{c}$ values correspond to a lengthened time scale over which coherent precessional motion of $\hat{S}_{z}(t)$ can develop, thus increasing its importance in the integrand of Eq. (5a). The width of the resonant contribution likewise depends strongly on the reduced correlation time. The theory developed above does not give $G_{ \pm}(t)$ in analytical form, but it seems clear from physical considerations discussed above that for $S=1$, where there is only a single oscillatory component of $\hat{S}_{z}(t)$, that the spectral density function of the resonant contribution has the form $j\left(\omega_{D}-2 \omega_{E} \pm \omega_{I}\right)$. In this case, the resonance is centered at $\omega_{D}=2 \omega_{E}$, and its width at half-height varies as $\tau_{c}^{-1}$. The calculations confirm that the resonance narrows as expected with increasing $\omega_{D} \tau_{c}$.

Figure 8 further illustrates the functional dependence of the longitudinal and transverse components to $R_{1 p}$. For this purpose the notation $R_{1 p}(\Theta, \Phi)$ has been employed, 


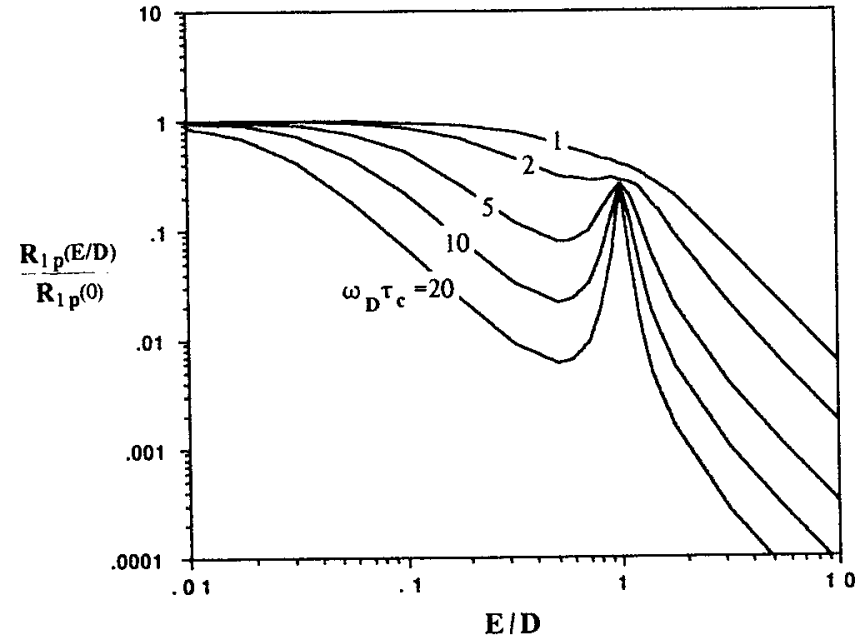

FIG. 7. Dependence of the dipolar NMR-PRE, normalized to $E / D=0$, on the reduced dipolar correlation time $\omega_{D} \tau_{C}$. The calculations assume $S=1, \Theta=0, \Phi=0$.

which specifies $R_{1_{p}}$ as a function of nuclear position $(\Theta, \Phi)$ in the molecular frame. $R_{1 p}$ is plotted in reduced form, $R_{1 p}(\Theta, \Phi) / R_{1 p}(0,0)$, as a function of $\Theta$ with $\Phi=0$. It is seen that for $E / D$ far from the resonance condition, i.e., $E / D \ll 1$ or $E / D \gg 1, R_{1 p}$ is largest at axial positions and smallest at equatorial positions. This behavior is characteristic of a uniaxial zfs and has been discussed in some detail previously. ${ }^{4,5}$ Physically, this behavior reflects the fact that, for the nonresonant contribution to $R_{1 p}$ (i.e., the contribution due to $\hat{S}_{z}$ ), the mean-squared local dipolar magnetic field due to $\hat{S}_{z}$ is larger at axial positions than at equatorial positions. For the uniaxial zfs limit (the curve $E / D=0$ in Fig. 8), this dependence is described mathematically by the

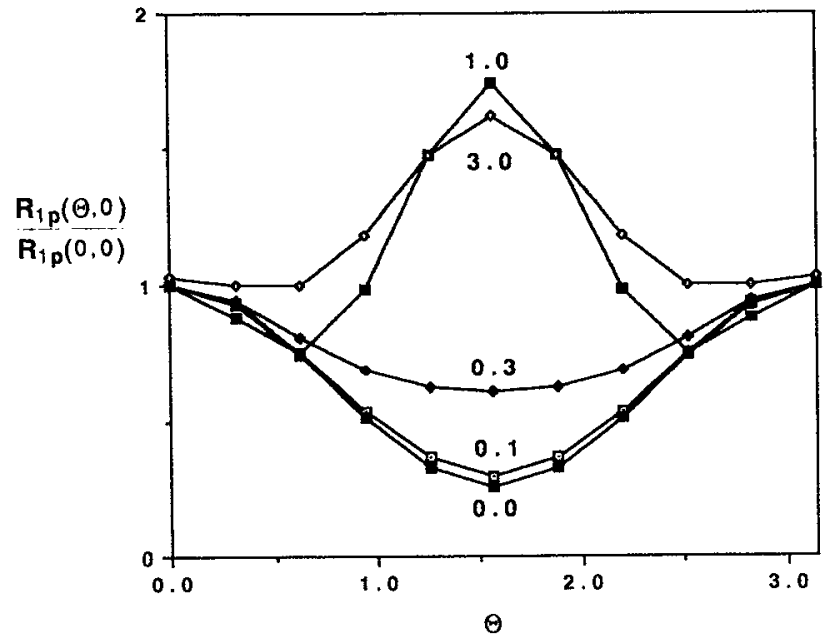

FIG. 8. Dependence of the dipolar $R_{1 p}$ on the polar angle $\Theta$ of the nuclear spin in the molecular coordinate frame. The curves assume $S=1, \omega_{D} \tau_{c}$ $=11.7, \Phi=0$, and the specified $E / D$ values. Relaxation enhancements are normalized to the calculated values at $\Theta=0, \Phi=0$.

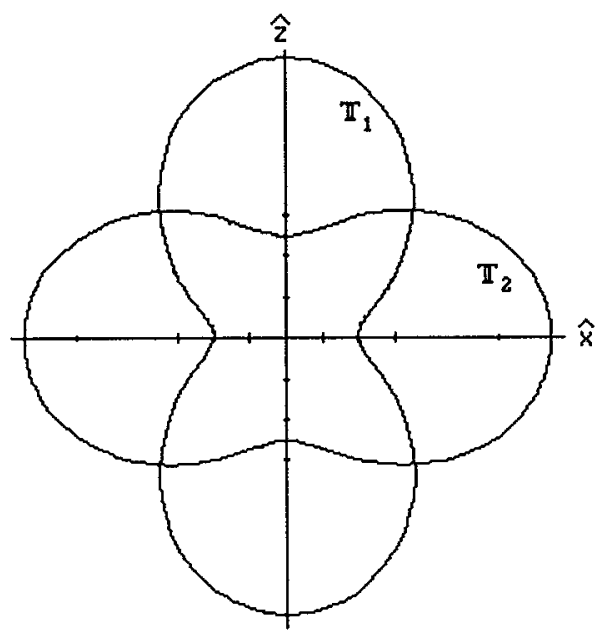

FIG. 9. Polar plots of the nonresonant $\left(T_{1}\right)$ and resonant $\left(\mathbb{T}_{2}\right)$ contributions to $R_{1 p}$. These plots were constructed from numerical calculations using Eqs. (19) $-(22)$ and (23c) with $S=1, E / D=1, \Phi=\pi / 4$.

function $\left[1+\hat{P}_{2}(\cos \Theta)\right]$ which appears in Eqs. $(20 \mathrm{~b})$ and (24).

The resonant contribution to $R_{1 p}$ [that due to the transverse spin components, $\left.\left\langle\hat{S}_{ \pm}(0) \cdot \hat{S}_{\mp}(t)\right\rangle\right]$ has the opposite $\Theta$ dependence, largest at equatorial positions and smallest at axial. In Fig. 8, this contribution is most pronounced for the curve with $E / D=1.0$. Physically, the functional dependence of the resonant contribution is a result of the local geometry of a mean-squared dipolar field that has its largest components at positions in the transverse plane, rather than along the molecular $\hat{z}$ axis. The resonant enhancement shown in Fig. 8 reflects this altered orientation.

The anisotropy in the NMR-PRE is illustrated by the polar plots of Fig. 9, which show the $\Theta$ variation of the nonresonant $\left(\mathbb{T}_{1}\right)$ and resonant $\left(\mathbb{T}_{2}\right)$ contributions to $R_{1 p}$ for $\Phi=\pi / 4$ [at this $\Phi$ value, the terms $\left(\mathbb{T}_{3}+\mathbb{T}_{3}^{*}\right)$ vanish]. Clearly the mean-squared magnetic fields that are associated with the nonresonant term are directed principally along the molecular $\hat{z}$ axis, while the mean-squared fields that are associated with the resonant term are largest in the transverse plane. Mathematically, the $\mathbb{T}_{1}$ and $\mathbb{T}_{2}$ ellipsoids are given by

$$
\begin{aligned}
& \mathbf{T}_{1} \propto\left[1+\hat{P}_{2}(\cos \Theta)\right], \\
& \mathbf{T}_{2} \propto\left[1-2^{-1} \hat{P}_{2}(\cos \Theta)\right] .
\end{aligned}
$$

The contributions of both $\mathbb{T}_{1}$ and $\mathbb{T}_{2}$ to $R_{1 p}$ are isotropic with respect to the molecular $\hat{x} \hat{y}$ plane.

The terms $\left(\mathbb{T}_{3}+\mathbb{T}_{3}^{*}\right)$ arise from $G_{ \pm}(t)$ and thus contribute to the resonant part of $R_{1 p}$. This sum gives rise to $R_{1 p}$ anisotropy in the $\hat{x} \hat{y}$ plane. Figure 10 shows the overall dependence of $R_{1 p}$ on $\Phi$ at various values of $E / D$, with $\Theta=\pi / 2$. Far from resonance $(E / D \ll 1$ or $E / D \gg 1)$, the reduced $R_{1 p}(\pi / 2, \Phi)$ is independent of $\Phi$. Physically, this describes the fact that the nonresonant contribution $\left(T_{1}\right)$ to $R_{1 p}$, which is associated with $G_{z}(t)$, is isotropic in the $\hat{x} \hat{y}$ plane. Near resonance, the terms that arise from $G_{ \pm}(t)$, namely, $\mathbb{T}_{2}$ and $\left(\mathbb{T}_{3}+\mathbb{T}_{3}^{*}\right)$, become important, and the lat- 


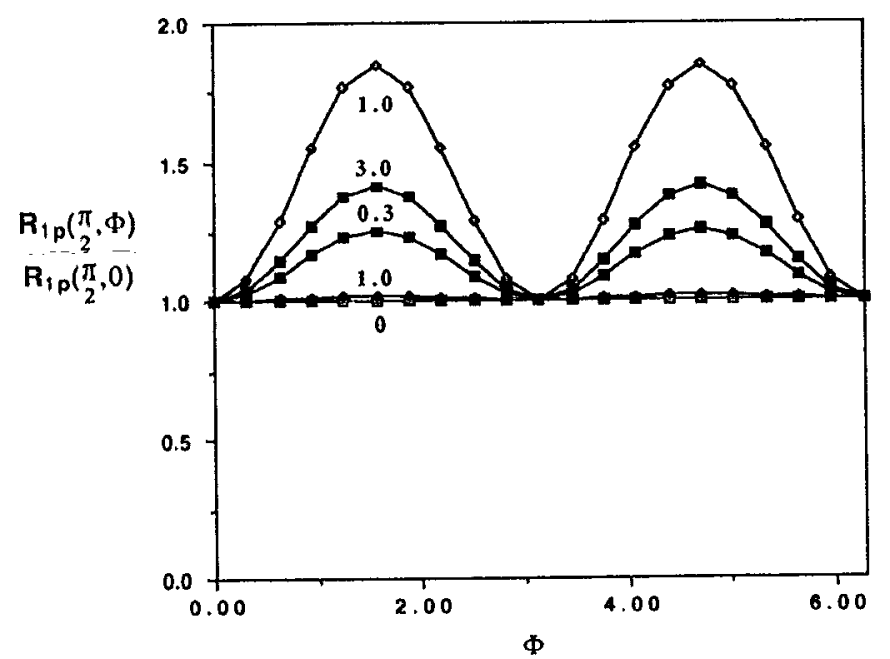

FIG. 10. Dependence of the dipolar $R_{i p}$ on the azimuthal angle $\Phi$ of the nuclear spin in the molecular coordinate frame. The curves assume $S=1$, $\omega_{D} \tau_{c}=11.7, \Theta=\pi / 2$, and the specified $E / D$ values. Relaxation enhancements are normalized to the calculated values at $\Theta=\pi / 2, \Phi=0$.

ter gives rise to the $\Phi$ anisotropy of $R_{1 p}$. Figure 11 shows polar plots of the resonant part of $R_{1 p}$ (that due to $\mathbb{T}_{2}+\mathbb{T}_{3}$ $\left.+T_{3}^{*}\right)$ in the $\hat{x} \hat{y}$ plane. The orientation of the $\Phi$ anisotropy depends on the sign of $E / D$. When $E / D>0$, the largest mean-squared fields associated with $\left(\mathrm{T}_{3}+\mathbb{T}_{3}^{*}\right)$ lie along the molecular $\hat{y}$ axis $(\Theta=\pi / 2, \Phi=\pi / 2)$; when $E / D>0$, they lie along the molecular $\hat{x}$ axis. The $\Phi$ anisotropy due to $\left(\mathbf{T}_{3}+\mathbf{T}_{3}^{*}\right)$ transforms as $\mp\left(\hat{x}^{2}-\hat{y}^{2}\right)$, the sign of the function depending on the $E / D$ ratio. The appropriate functional dependence of the sum is illustrated in Fig. 12.

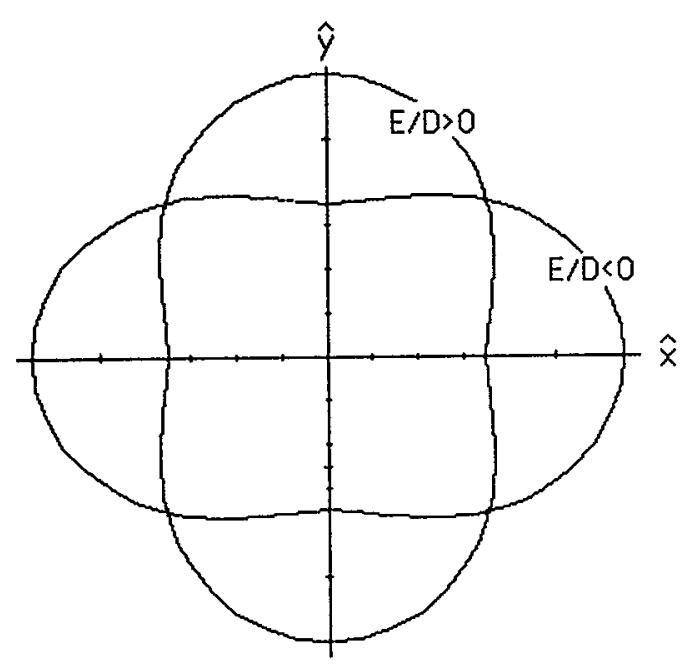

FIG. 11. Polar plots of the variation of the resonant part of $R_{1 p}$ (that due to $\mathbf{T}_{2}+\mathbf{T}_{3}+\mathbf{T}_{3}^{*}$ ) in the molecular $\hat{x} \hat{y}$ plane. The plots are based on numerical calculations using Eq. (19) with $S=1, E / D=1$, and the $\mathbb{T}_{1}$ term ignored. The two $T_{2}$ ellipsoids correspond to positive and negative values of the $E / D$ ratio.

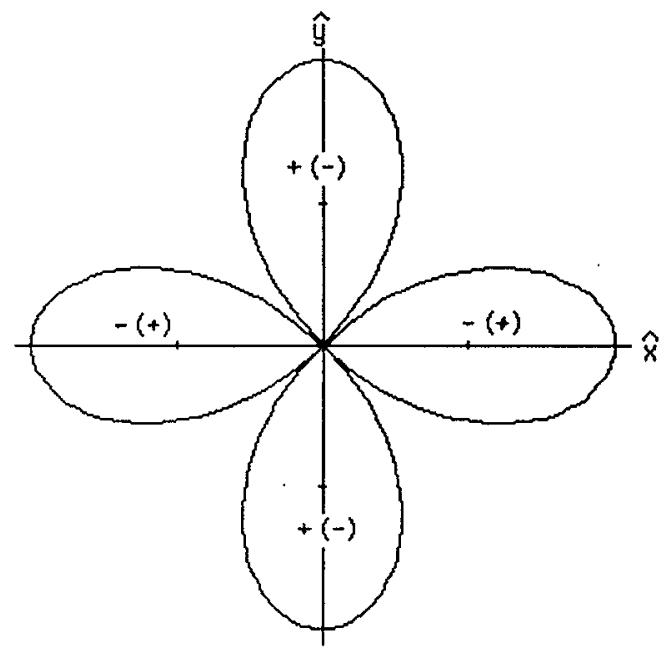

FIG. 12. Polar plot of the variation of the anisotropic part of $R_{1 p}$ (that due to $\left.T_{3}+T_{3}^{*}\right)$ in the molecular $\hat{x} \hat{y}$ plane. The signs correspond to positive (negative) values of $E / D$.

\section{DISCUSSION}

In summary, the presence of anisotropy in the zfs tensor profoundly affects the NMR-PRE in the zfs limit. This influence results physically from the effects of the offdiagonal terms of $\mathscr{H}_{\text {zfs }}$ on the motion of $\hat{S}_{z}(t)$, which, in the presence of zfs anisotropy, undergoes precessional motion about an axis perpendicular to $\hat{z}$. This motion can lead to a profound decrease in the low frequency components of the time correlation function $G_{z}(t)$. These components are normally responsible for the largest part of $R_{1 p}$, and thus the presence of zfs anisotropy acts to lessen the magnitude of the NMR-PRE. A second related phenomenon occurs when the precessional motion of $\hat{S}_{z}(t)$ comes into resonance with the precessional motion of the transverse components of $S$. This resonance can produce a static (or low frequency) component of $S$ in the transverse plane, which can provide a highly efficient relaxation pathway within a relatively narrow range of $E / D$ ratios.

The calculations presented above were carried out for $S=1$. Further studies are needed to determine whether parallel phenomena occur for other spin values. For $S>1$, the effect of terms in $\mathscr{H}_{S}$ which join states of $\Delta m_{S}= \pm 2$ will create precessional oscillations in $\hat{S}_{z}$ thereby shortening $G_{z}(t)$ and decreasing $R_{1 p}$ in a manner that is similar to that described above for $S=1$. However, the precessional motions will not simply be sinusoidal, but rather more complicated functions of time. Resonances in the transverse components of the magnetization are likewise expected to occur for $S>1$. However, the detailed functional dependence of the resonances on $E / D$ will certainly differ from that for $S=1$ in a manner that depends on the details of the level crossings of the spin system. The $R_{1 p}$ behavior that is characteristic of different spin values can readily be calculated from the theory of Sec. III, which is valid for all $S$.

Importance of zfs anisotropy on the practical analysis of NMR-PRE data. The effects of zfs anisotropy have rather 
profound implications for the analysis of NMR-PRE data in the zfs limit. When the zfs is isotropic ( $D \neq 0, E=0$ ), the magnitude of the NMR-PRE is nearly independent of the magnitude of the $\mathrm{zfs}$ splitting. This is true because the $\mathrm{zfs}$ splitting parameter $D$, which determines the transition frequencies of the $S$ spin system, affects only the high frequency terms of $R_{1 p}$ [those proportional to $j\left(\omega_{\mu} \pm \omega_{I}\right)$ ], not the low frequency term [that proportional to $j\left(\omega_{I}\right)$ ]. However, it is the low frequency term that is normally responsible for the largest part of the NMR-PRE. The high frequency terms are small when $\omega_{D} \tau_{c}>1$, which is very frequently the situation in the $\mathrm{zfs}$ limit. Also, I have shown previously that when $\omega_{D} \tau_{c}<1$, the NMR-PRE is well described by Zeeman limit theory, even in situations where $\mathscr{H}_{\text {zfs }}>\mathscr{H}_{Z}$. Thus the magnitude and properties of the NMR-PRE in the uniaxial zfs limit are largely independent of the specific numerical value of $D$.

The presence of a uniaxial zfs has a profound effect on the NMR-PRE in the zfs limit due to the effect of $\mathscr{H}_{\text {zfs }}$ in determining the quantization axes of the spin precessional motion (molecule-fixed vs laboratory). However, in the zfs limit, the magnitude of the uniaxial part of $\mathscr{H}_{\text {zfs }}$ has little influence on the $R_{1 p}$. In contrast, the anisotropic part of $\mathscr{H}_{\text {zfs }}$ induces precessional motion in $\hat{S}_{z}$ which can, as described above, dramatically shorten the time correlation function $G_{z}(t)$, thereby lowering the magnitude of the PRE. This effect is important when $2 \omega_{E} \tau_{c}>1$ (for $S=1$ ), and its magnitude is quite sensitive to the specific value of $E$.

It should be emphasized that the theory developed here assumes that the spin system is in the zfs limit and neglects entirely effects of the external magnetic field on the motion of $\hat{S}$. These assumptions are appropriate for the present purpose, which is to understand the physical phenomena that are associated with zfs anisotropy and its influence on the NMR-PRE. However, an external magnetic field that has components perpendicular to the principal axis of the zfs tensor may well have important effects on the spin energy levels in the vicinity of the level crossings. This is true in the zfs limit as well as in the intermediate and Zeeman regimes. Thus it seems likely that the quantitative behavior of the resonant component of $R_{1 p}$ may be significantly altered by a static magnetic field even when $\mathscr{H}_{Z}<\mathscr{H}_{\text {zfs. }}$. However, the inclusion of $\mathscr{H}_{Z}$ in the theory of Sec. III adds substantially to the mathematical complexity of the analysis and has not yet been attempted.

Experimental investigations that are sufficiently detailed to examine systematically the phenomena described in Sec. IV are not currently available in the literature. Such studies are less straightforward to carry out than for the Zeeman limit, where the spin energy levels can be varied continuously as a function of external magnetic field strength. In the zfs limit, the level spacings are determined primarily by the zfs parameters $D$ and $E$, which depend on the chemical environment of the metal ion and cannot be continuously varied. It is possible that a useful variation in the $E / D$ ratio could be achieved through a careful choice of chemical system, for example, in a series of asymmetrically substituted metalloporphyrins. In such a study, com- panion static magnetic susceptibility measurements of $D$ and $E$ would be needed. For one Mn(III)-porphyrin complex, that of $\mathrm{Mn}$ (III) with tetraphenylsulfanyl porphyrin, ${ }^{29}$ the magnetic field dispersion profiles of the NMRPRE exhibit unique qualitative features that are highly interesting though poorly understood. The $\mathrm{zfs}$ is known to be rather large for $\mathrm{Mn}$ (III) porphyrins, ${ }^{30,31}$ and the zfs interaction undoubtedly exerts a major influence on the behavior of the NMR-PRE. This and other porphyrin complexes may in the future provide useful model systems for studying the phenomena described here, but at present the data base is insufficient for this purpose.

Other experimental approaches can also be suggested. For example, the $\Theta$ and $\Phi$ anisotropies of $R_{1 p}$ could be probed through the study of chemically distinct nuclear sites within a given complex ion of known molecular structure. To be useful in the present context, this approach requires an accurate knowledge of molecular structure as well as companion measurements of the zfs parameters $D$ and $E$. Thus the construction of a data base that is useful for systematic examination of the phenomena described in Sec. IV will require considerable care in experimental design and in the selection of appropriate chemical systems.

\section{APPENDIX}

We wish to show that the trace

$$
\begin{aligned}
\operatorname{Tr}\left\{S_{p}^{(1)}(t) S_{p^{\prime}}^{(1)}(0)\right\}= & \operatorname{Tr}\left\{\exp \left(i \mathscr{H}_{S} t\right) S_{p}^{(1)}\right. \\
& \left.\times \exp \left(-i \mathscr{H}_{S} t\right) S_{p^{\prime}}^{(1)}\right\}
\end{aligned}
$$

with $\mathscr{H}_{S}$ given by Eq. (1c) vanishes unless $p+p^{\prime}$ is even. Writing the exponential factors as series,

$\exp \left(i \mathscr{H}_{s} t\right)=\sum_{n=0}^{\infty} \frac{\left\{i\left[(2 / 3)^{1 / 2} D S_{0}^{(2)}+E\left(S_{+2}^{(2)}+S_{-2}^{(2)}\right)\right] t\right\}^{n}}{n !}$

and expanding each product in Eq. (A1) gives an infinite sum of terms. Each term in the sum has the form

$$
T=G(t)\left(\prod_{j} S_{p_{j}}^{(2)}\right) S_{p}^{(1)}\left(\prod_{j^{\prime}} S_{p_{j^{\prime}}}^{(2)}\right) S_{p^{\prime}}^{(1)},
$$

where the individual spin operators are written explicitly and $p_{j}, p_{j^{\prime}}= \pm 2,0$, and $p, p^{\prime}= \pm 1,0$. The $S_{r}^{(q)}$ are spherical tensor operators and transform under rotations as the spherical harmonics $Y_{q, r}(\beta, \alpha)$ of the corresponding rank and order. The spatial averages of the terms $T$ are required, and these are most readily evaluated by writing the spherical harmonics in equivalent form as special cases of the Wigner rotation matrix elements,

$$
y_{q, m}(\beta, \alpha)=\left(\frac{2 q+1}{4 \pi}\right)^{1 / 2} \mathscr{D}_{0, m}^{(q)}(\alpha, \beta, \gamma) .
$$

Thus each pairwise product $S_{r}^{(q)} S_{r^{\prime}}^{\left(q^{\prime}\right)}$ transforms as the product 


$$
\begin{aligned}
Y_{q, r}(\beta, \alpha) Y_{q^{\prime}, r^{\prime}}(\beta, \alpha)= & \left(\frac{1}{4 \pi}\right)(2 q+1)^{-1 / 2}\left(2 q^{\prime}+1\right)^{-1 / 2} \\
& \times \mathscr{D}_{0, r}^{(q)}(\alpha \beta \gamma) \mathscr{D}_{0, r^{\prime}}^{\left(q^{\prime}\right)}(\alpha \beta \gamma) .
\end{aligned}
$$

Contracting the product of rotation matrix elements by the Clebsch-Gordan series ${ }^{27}$ gives

$$
\begin{aligned}
\mathscr{D}_{r, 0}^{(q)} \mathscr{D}_{r^{\prime}, 0}^{\left(q^{\prime}\right)}= & (-1)^{r+r^{\prime}} \sum_{q^{\prime \prime}}\left(2 q^{\prime \prime}+1\right) \\
& \times\left(\begin{array}{ccc}
q & q^{\prime} & q^{\prime \prime} \\
r & r^{\prime} & -\left(r+r^{\prime}\right)
\end{array}\right) \\
& \times\left(\begin{array}{ccc}
q & q^{\prime} & q^{\prime \prime} \\
0 & 0 & 0
\end{array}\right) \mathscr{D}_{r+r^{\prime}, 0}^{\left(q^{\prime \prime}\right)} .
\end{aligned}
$$

This product vanishes unless the triangle condition $\Delta\left(q, q^{\prime}, q^{\prime \prime}\right)$ is satisfied, and unless the sum of the orders of the rotation matrix elements on the left equals the order of the matrix element on the right. By repetitive pairwise contraction of the spin operators, each product (A3) can be contracted to a term containing a single spin operator $S_{\sigma}^{(\rho)}$, for which the order $\sigma$ is

$$
\sigma=\sum_{j, j^{\prime}} p_{j}+\sum_{j^{\prime}} p_{j^{\prime}}+p+p^{\prime}
$$

The trace over the spin variables, $\operatorname{Tr}\left\{S_{\sigma}^{(\rho)}\right\}$, vanishes unless $\rho=\sigma=0$. From Eq. (A6) and the fact that $p_{j}$ and $p_{j^{\prime}}$ are even, this implies that the trace of Eq. (A1) vanishes unless $p+p^{\prime}$ is even.

'I. Solomon, Phys. Rev. 99, 559 (1955).

${ }^{2}$ N. Bloembergen, J. Chem. Phys. 27, 572, 595 (1957).

${ }^{3}$ N. Bloembergen and L. O. Morgan, J. Chem. Phys. 34, 842 (1961).
${ }^{4}$ R. A. Dwek, NMR in Biochemistry (Oxford University, Oxford, 1971).

${ }^{5}$ R. R. Sharp, J. Chem. Phys. 98, 912 (1993).

${ }^{6}$ R. R. Sharp, J. Chem. Phys. 98, 2507 (1993).

${ }^{7}$ R. R. Sharp, J. Chem. Phys. 93, 6921 (1990).

${ }^{8}$ T. Bayburt and R. R. Sharp, J. Chem. Phys. 92, 5892 (1990).

${ }^{9}$ R. R. Sharp, J. Magn. Reson. 100, 491 (1992).

${ }^{10}$ N. Benetis, J. Kowaleski, L. Nordenskiold, H. Wennerstrom, and P.-O. Westlund, Molec. Phys. 48, 329 (1983).

${ }^{11}$ N. Benetis and J. Kowaleski, J. Magn. Reson. 65, 13 (1985).

${ }^{12}$ N. Benetis, J. Kowalewski, L. Nordenskiold, H. Wennerstrom, and P.-O. Westlund, Mol. Phys. 50, 515 (1983).

${ }^{13}$ U. Lindner, Ann. Phys. (Leipzig) 16, 319 (1965).

${ }^{14}$ H. L. Friedman, M. Holz, and H. G. Hertz, J. Chem. Phys. 70, 3369 (1979).

${ }^{15}$ U. Lindner, Ann. Phys. (Leipzig) 16, 319 (1965).

${ }^{16}$ D. T. Pegg and D. M. Doddrell, Aust. J. Chem. 31, 475 (1978).

${ }^{17}$ D. T. Pegg, D. M. Doddrell, M. R. Bendall, and A. K. Gregson, Aust. J. Chem. 29, 1885 (1976).

${ }^{18}$ H. L. Friedman, M. Holz, and H. G. Hertz, J. Chem. Phys. 70, 3369 (1979).

${ }^{19}$ T. R. Chen, S.-J. Den, and L.-P. Hwang, Proc. Natl. Sci. Counc. (Rep. of China) (A) 8, 224 (1984).

${ }^{20}$ L.-P. Hwang and C.-Y. Ju, J. Chem. Phys. 83, 3775 (1985).

${ }^{2 t}$ P.-L. Wang, J.-H. Lee, S.-M. Huang, and L.-P. Hwang, J. Magn. Reson. 73, 277 (1987).

${ }^{22}$ N. Benetis, J. Kowalewski, L. Nordenskiold, H. Wennerstrom, and P.-O. Westlund, Mol. Phys. 48, 329 (1983).

${ }^{23}$ P.-O. Westlund, H. Wennerstrom, L. Nordenskiold, J. Kowalewski, and N. Benetis, J. Magn. Reson. 59, 91 (1984).

${ }^{24}$ N. Benetis and J. Kowaleski, J. Magn. Reson. 65, 13 (1985).

${ }^{25}$ 1. Banci, I. Bertini, F. Briganti, and C. Luchinat, J. Magn. Reson. 66, 58 (1986).

${ }^{26}$ S. Szymanski, A. M. Gryff-Keller, and G. Binsch, J. Magn. Reson. 68, 399 (1986).

${ }^{27}$ B. L. Silver, Irreducible Tensor Methods (Academic, New York, 1976).

${ }^{28}$ A. D. McLachlan, Proc. R. Soc. London, Ser. A 280, 271 (1964).

${ }^{29}$ S. H. Koenig, R. D. Brown III, and M. Spiller, Magn. Reson. Med 4, 252 (1987).

${ }^{30}$ D. V. Behere and S. Mitra, Inorg. Chem. 19, 992 (1980).

${ }^{31}$ D. V. Behere, V. R. Marathe, and S. Mitra, Chem. Phys. Lett. 81, 57 (1981). 